\title{
Vortioxetine in the treatment of adult patients with major depressive disorder: a meta-analysis of randomized double-blind controlled trials
}

\author{
Asres Berhan ${ }^{1 *}$ and Alex Barker ${ }^{2}$
}

\begin{abstract}
Background: Vortioxetine is a novel multimodal compound that has recently been approved by the FDA for the treatment of major depressive disorder (MDD). It is a selective serotonin (5-HT) 3A and 5-HT7 receptor antagonist, 5-HT1B receptor partial agonist, 5-HT1A receptor agonist and inhibitor of serotonin transporters. The objective of this meta-analysis was to evaluate the efficacy and safety of vortioxetine in adults with MDD.

Methods: A literature search was conducted in the databases of PubMed, EMBASE, Cochrane library and HINARI. The meta-analysis was conducted by including randomized controlled trials that assessed the efficacy and safety of vortioxetine in adult patients with MDD. Using the random effects model, which assumes individual studies are estimating different treatment effects, the efficacy and safety of vortioxetine was determined by weighted mean differences (WMDs) and odds ratios (ORs). The findings were considered as statistically significant when the 95\% Cl of WMDs and ORs did not include 0 and 1, respectively. Heterogeneity testing, meta-regression and sensitivity analysis were also performed.

Results: During the initial literature search about 151 publications were identified. Based on the predetermined inclusion criteria, 7 randomized controlled trials were included. The pooled analysis demonstrated a statistically significant reduction in the Montgomery-Åsberg Depression Rating Scale (MADRS) total score from baseline among patients who were on vortioxetine (WMD $=-3.92 ; 95 \% \mathrm{Cl},-5.258$ to -2.581$)$. Furthermore, a statistically significant number of patients with MDD who were on vortioxetine have achieved a greater than or equal to $50 \%$ reduction in depression symptoms from baseline. However, a significant number of patients who were on vortioxetine therapy reported more adverse events than patients who were on placebo (overall $\mathrm{OR}=1.21 ; 95 \% \mathrm{Cl}, 1.06$ to 1.38).

Conclusions: Therapy with vortioxetine was significantly associated with reduction in depression symptoms from baseline compared to placebo. Nevertheless, a significant number of patients who were on vortioxetine therapy have reported more adverse events.
\end{abstract}

Keywords: Vortioxetine, Major depressive disorder, Meta-analysis, Serotonin, Depression symptoms

\section{Background}

MDD is one of the leading causes of disability worldwide; it is highly recurrent and the symptoms usually persist for longer period. Despite the availability of many antidepressants (tricyclic antidepressants, monoamine oxidase inhibitors, 5-HT reuptake inhibitors and serotonin and norepinephrine reuptake inhibitors) with different

\footnotetext{
* Correspondence: asresberhan@gmail.com

${ }^{1}$ Hawassa University College of Medicine and Health Sciences, P. O. Box: 1560, Hawassa, Ethiopia

Full list of author information is available at the end of the article
}

mode of actions, an unmet need exists in treating suboptimal efficacy, remission rate and cognition impairments [1]. Therapy with the currently available antidepressant is also associated with side effects, such as sexual dysfunction, suicide risk and weight gain [2,3]. Furthermore, in young adults, long-term antidepressant use suggested to be associated with an increased risk of type 2 diabetes mellitus and bleeding risk $[4,5]$.

Vortioxetine is a novel multimodal compound that has recently been approved by the Food and Drug Administration (FDA) of the United States of America for the 
treatment of MDD. This drug is a 5-HT 3A, 5-HT7 and 5HT1D receptor antagonist, 5-HT1B receptor partial agonist, 5-HT1A receptor agonist and inhibitor of the serotonin transporter [6,7]. Vortioxetine showed an extended absorption, a medium clearance, a large volume of distribution and a relatively long elimination half-life in healthy young volunteers [8]. Concomitant therapy with drugs involved in the CYP P450 pathways does not seem to have a statistically significant interactions with vortioxetine [9].

Randomized controlled trials that assessed the efficacy of vortioxetine for the treatment of patients with MDD reported contradictory findings. The efficacy of vortioxetine in a study with a duration of 6 weeks was significantly superior to placebo [10]. Similarly, 3 randomized controlled trials with a duration of 8 weeks reported a significant reduction in depression symptoms [11-13]. However, vortioxetine did not differ significantly from placebo in reducing depression symptoms in one study with a duration of 6 weeks and in two studies with durations of 8 weeks [14-16]. The adverse events reported by the patients in the randomized trials were also inconclusive. Thus the primary aim of this meta-analysis is to evaluate the efficacy and safety of vortioxetine at different doses in the treatment of MDD by including randomized controlled trials.

\section{Methods}

\section{Search strategy}

Literature search was conducted by both authors in the databases of PubMed, EMBASE, Cochrane library, HINARI and Google scholar. Major publishers' websites (Elsevier Science-Science Direct, Nature Publishing Group, Oxford University Press, PsycARTICLES, Science and Wiley-Blackwell) were searched via HINARI. Our search was further strengthened by searching the reference lists of retrieved articles.

The selected key search terms were: MDD, vortioxetine (Lu AA21004), MADRS, Hamilton Rating Scale for Depression (24 items) (HAM-D24) and adverse events. During searching, the term vortioxetine or Lu AA21004 was alternatively combined with other search terms with the help of Boolean logic (AND, OR and NOT).

\section{Inclusion criteria and study selection}

The predetermined study inclusion criteria were: 1) randomized controlled trials that assessed the efficacy and safety of vortioxetine in adult patients with MDD, and studies that recruited patients with MDD presenting with a current major depressive episode of at least 4 weeks duration with no other concurrent psychiatric disorders and MADRS total score of not less than 22 at screening and baseline visits; 2) studies that reported one of the following efficacy or safety measures: change in MADRS and/or in HAM-D24 total score from baseline, proportion of patients who achieved a $\geq 50 \%$ MADRS and/or HAMD24 score reduction from baseline, and number of patients with adverse events.

Study selection was conducted by both authors independently in two stages: first, the abstracts of all the retrieved articles were reviewed and the studies were grouped as either "eligible for full document review" or "ineligible for full document review". Then, after full document review of all studies that were grouped as "eligible for full document review" were grouped as either "eligible for meta-analysis" or "ineligible for meta-analysis".

\section{Data extraction and study quality assessment}

The data extraction was also conducted independently by both authors with a similar data extraction template. From the included studies the following information was abstracted: name of the first author, year of publication, duration of therapy, dose, sample sizes, change in MADRS and HAM-D total score from baseline, the number of patients with $\geq 50 \%$ decrease in MADRS and HAM-D total score, number of remitters and number of patients with adverse events.

Risk of bias among the included studies was assessed with the Cochrane risk of bias assessment tools. The key domains were: random sequence generation, allocation concealment, blinding of participants and personnel, blinding of outcome assessment, incomplete outcome data, selective reporting and other bias.

\section{Data synthesis and statistical analysis}

In studies where the standard error (SE) and p-values were reported, for ease of use, we transformed to equivalent standard deviation values. Using the random effects model, the efficacy and safety of vortioxetine was determined with WMDs and ORs. For continues variables (the change in MADRS and HAM-D total score from baseline) the WMDs and 95\% confidence intervals (CI) were computed using the inverse variance method. While, the ORs were computed using the Mantel-Haenszel $(\mathrm{M}-\mathrm{H})$ method. The findings were considered as statistically significant when the $95 \%$ CI of WMDs and ORs did not include 0 and 1 , respectively. Due to lack of data and inconsistency of reported adverse events across the included studies, the meta-analysis to assess the association of vortioxetine with specific adverse events was restricted to two variables (nausea and hyperhidrosis).

The heterogeneity among the included studies was assessed with $\mathrm{I}^{2}$ statistics; when the value of $\mathrm{I}^{2}$ was greater than or equal to $50 \%$ it was considered as statistically significant. To assess the possible sources of heterogeneity, subgroup analysis based on the durations of therapy and meta-regression using vortioxetine dose as a 
covariate were performed. To assess the stability of the pooled values to outliers, sensitivity analyses (leave one study out at a time) were performed. All the statistical analyses were performed using OpenMeta-analyst software [17].

\section{Results}

Using the Google scholar search engine, for the term vortioxetine about 151 publications were identified. Based on the titles proximity to the objective of this study 66 articles were retrieved. In this meta-analysis, in line with the predetermined inclusion criteria, 7 randomized controlled trials were included [10-16] (Figure 1). Except one study [15], all the included studies assessed the efficacy and safety of vortioxetine by randomizing patients in to different study arms with different doses. Reasons for the exclusion of studies were: in vitro studies, animal studies, trial with healthy volunteers and review articles. As presented in Table 1, five of the included studies used duloxetine $[11,12,14,16]$ or venlafaxine [10] as an active reference. While, five of the studies have a duration of therapy of 8 weeks $[11-14,16]$; the remaining two studies have a duration of therapy of 6 weeks $[10,15]$. In the selected studies 2,099 patients with MDD were on vortioxetine; 1,130 were on placebo; 705 were on other active drugs (duloxetine or venlafaxine). Though the bias risk assessment demonstrated low risk of bias in randomization and blinding, the presence of other biases (recruitment bias, biases related to the clinical settings etc.) cannot be ruled out.

Figure 2 shows the MADRS mean change from baseline. At the end of interventions, there was a statistically significant reduction in the MADRS total score from baseline among patients who were on vortioxetine as compared with placebo treated patients (WMD $=-3.92$; 95\% CI, -5.258 to -2.581 ). Though the overall WMD demonstrated the significant reduction in the MADRS total score from baseline in vortioxetine treated groups, in two of the studies the change was not significantly different from placebo treated patients $[14,15]$. On the other hand, heterogeneity testing revealed the presences of significant heterogeneity among the included studies $\left(\mathrm{I}^{2}=68 \%\right)$. The meta-regression using doses of vortioxetine as covariate showed a statistically significant reduction in MADRS total score with patients who used higher doses of vortioxetine (slope $=-0.031 ; 95 \% \mathrm{CI},-0053$ to $-0.009 ; \mathrm{P}=0.005$ ) (Figure 3 ). While, the subgroup analysis based on the duration of therapy did not demonstrate a significant difference in therapeutic outcome. Moreover, the sensitivity analysis showed the stability of the overall WMD (WMD do not change significantly when any of the studies is excluded from the analysis).

As shown in Figure 4, the odds of vortioxetine treated patients achieving a $\geq 50 \%$ MADRS score reduction from baseline was about 3 times higher than placebo treated patients (Overall OR $=2.87$; 95\% CI, 2.39 to 3.44). Treatment with vortioxetine was significantly associated with a mean decline in HAM-D24 total score from baseline (WMD $=-2.67 ; 95 \% \mathrm{CI},-3.96$ to -1.38 ). Consistently, the heterogeneity testing showed the presence of significant inconsistencies among the included studies $\left(\mathrm{I}^{2}=65 \%\right)$. The odds of patients with a decrease in $\geq 50$ HAM-D24 total score from baseline was more than 2 fold as compared to placebo treated patients (overall $\mathrm{OR}=2.14$; 95\% CI, 1.54 to 2.97 ).

As shown in Figure 5, comparison based on treatment related adverse events demonstrated that a significantly large number of patients treated with vortioxetine experienced more adverse events than patients who were on placebo (overall $\mathrm{OR}=1.21 ; 95 \% \mathrm{CI}, 1.06$ to 1.38 ). The odds of patients who experienced nausea was about 3 fold higher among patients who were on vortioxetine as compared to placebo treated patients (overall $\mathrm{OR}=2.89$; $95 \% \mathrm{CI}, 2.40$ to 3.48 ). While, the number of patients who were on vortioxetine and experienced hyperhidrosis was

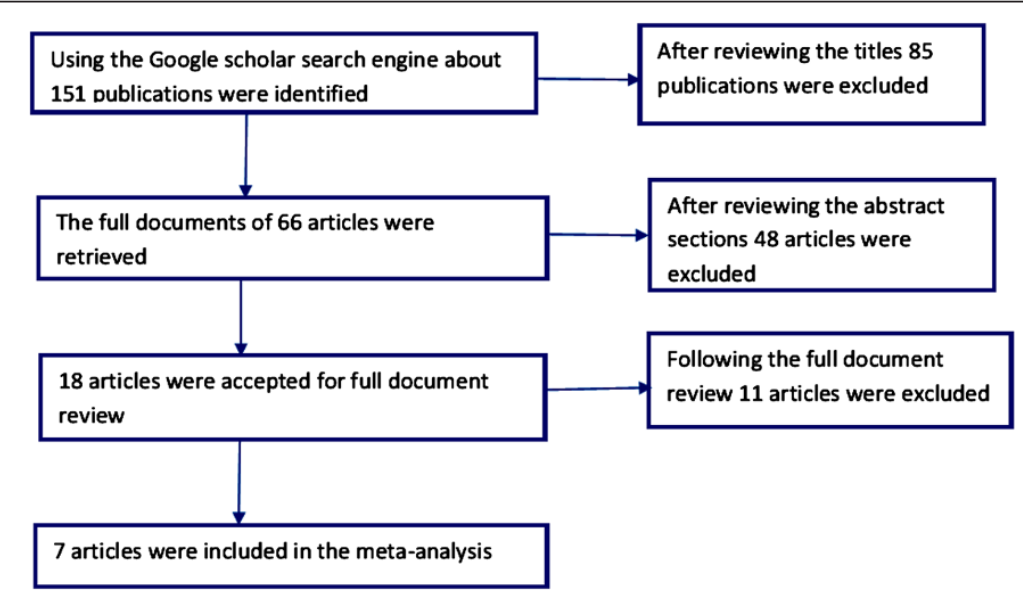

Figure 1 Flow diagram showing studies selection. 
Table 1 Summary of the included studies in the meta-analysis

\begin{tabular}{|c|c|c|c|c|c|c|c|}
\hline Authors & Year & Duration & Group1/Sample & Group 2/Sample & Group 3/Sample & Group 4/Sample & Group 5/Sample \\
\hline Alvarez E et al. [10] & 2011 & 6 weeks & Vortioxetine $5 \mathrm{mg} / 108$ & Vortioxetine $10 \mathrm{mg} / 100$ & Venlafaxine/113 & Placebo/105 & --- \\
\hline Baldwin DS et al. [14] & 2012 & 8-week & Vortioxetine 2.5 mg/155 & Vortioxetine 5 mg/155 & Vortioxetine $10 \mathrm{mg} / 151$ & Duloxetine 60 mg/149 & Placebo/145 \\
\hline Mahableshwarkar AR et al. [16] & 2013 & 8 weeks & Vortioxetine 2.5 mg/146 & Vortioxetine 5 mg/153 & Duloxetine 60 mg/149 & Placebo/149 & --- \\
\hline Katona C et al. [1 1] & 2012 & 8 weeks & Vortioxetine $5 \mathrm{mg} /$ day/154 & Duloxetine/147 & Placebo/145 & --- & --- \\
\hline Jain R et al. [15] & 2013 & 6 wks & Vortioxetine 5 mg/300 & Placebo/300 & --- & --- & --- \\
\hline Boulenger JP et al. [12] & 2013 & 8 weeks & Vortioxetine 15 mg/151 & Vortioxetine 20 mg/151 & Duloxetine/147 & Placebo/158 & --- \\
\hline Henigsberg $\mathrm{N}$ et al. [13] & 2012 & 8 wks & Vortioxetine 1 mg/124 & Vortioxetine 5 mg/129 & Vortioxetine $10 \mathrm{mg} / 122$ & Placebo/128 & --- \\
\hline
\end{tabular}




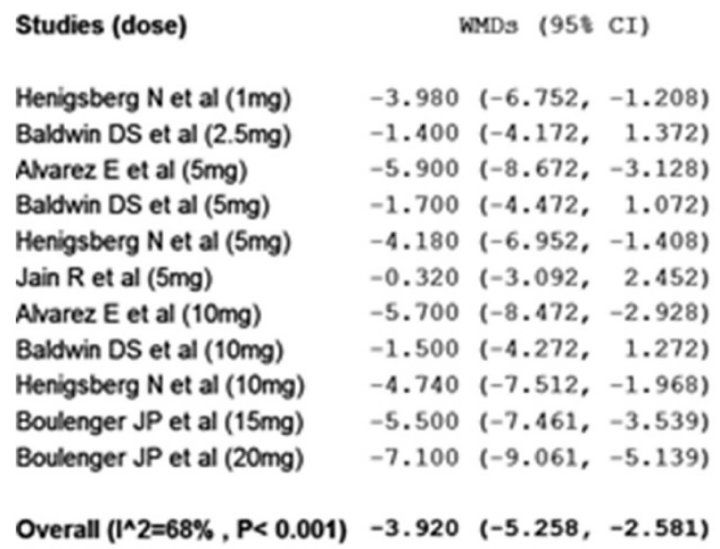

$$
\text { O }
$$$$
\text { Figu }
$$

Figure 2 Weighted mean difference of the change in MADRS total score from baseline.

no statistically different from placebo treated patients (overall OR $=1.39 ; 95 \% \mathrm{CI}, 0.72$ to 2.66 ).

\section{Discussion}

This meta-analysis demonstrated the efficacy of vortioxetine in reducing depression symptoms in adult patients with MDD. Based on both depression rating scales (MADRS and HAM-D24) a significant number of patients with MDD who were on vortioxetine therapy have achieved a greater than or equal to $50 \%$ depression symptom reduction from baseline. The decrease in depression symptoms seems to be intensified with an increase in the dose of vortioxetine. Furthermore, the efficacy of vortioxetine did not appear to decrease with a long term use; in two multicenter, open-label, flexible-dose extension studies the efficacy of vortioxetine in the treatment of MDD was maintained for about 12 months $[18,19]$. One of the limitations of most currently available antidepressants is the delay to induce either response or remission (slow onset of action). The lag in onset of antidepressant action is associated with negative consequences, such as increased suicide risk and other deliberate self-harm [20]. However, in vortioxetine treated groups, a significant number of patients with MDD has achieved response or remission within 6 to 8 weeks.

Heterogeneity testing revealed the presence of significant inconsistency among the included studies. While without improvement in the heterogeneity, the sensitivity analysis (leave one study out at a time analysis) showed the stability of the overall WMD. Yet, the reliability of the findings about the efficacy of vortioxetine from this metaanalysis does not seem diminished. This is because; when the number of the included studies in a meta-analysis is small and the heterogeneity is large, the robustness of the finding is best assessed with sensitivity analysis [21].

On the other hand, as compared to placebo treated patients, therapy with vortioxetine was significantly associated with the reported sum total adverse events. Among vortioxetine treated patients with MDD, a statistically significant number of them had experienced nausea. Though

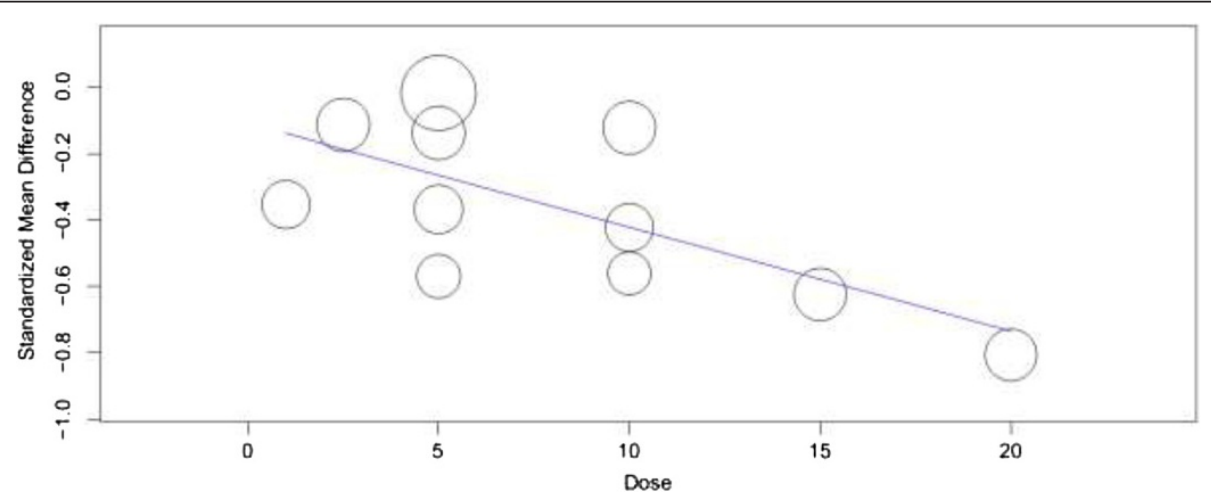

Figure 3 Change in mean difference in MADRS total score by dose of vortioxetine. 


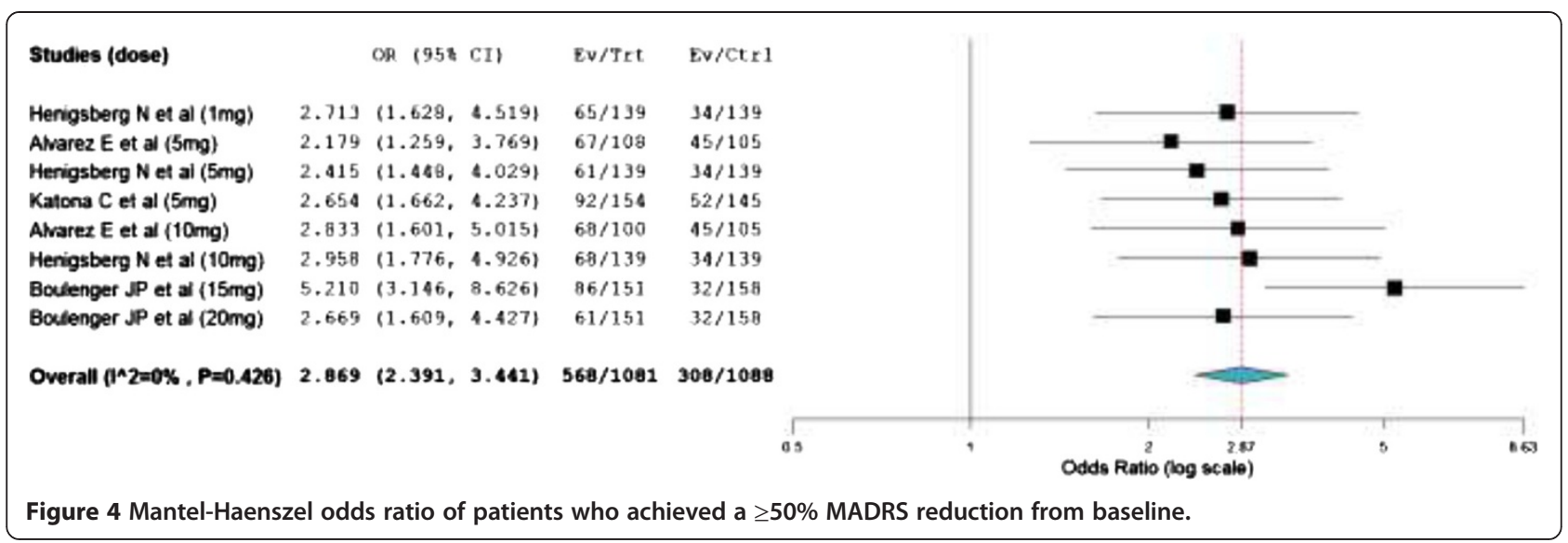

primary studies reported the association of vortioxetine with hyperhidrosis, the number of patients with an increased incidences of sweating was not different from placebo treated patients in this meta-analysis. On the other hand, a systematic review that describes the efficacy and safety of vortioxetine concluded no clinically-relevant weight change and suicidal ideation or behavior differences between vortioxetine treated and placebo treated [22]. Moreover, a randomized controlled trial that was designed to assess the effect of single or multiple doses of vortioxetine on cognitive or psychomotor performance concluded no impairment in psychomotor performance [23].

As limitations, first, this meta-analysis noted a significant heterogeneity among the included studies. The most likely explanations for the inconsistency across the included studies could be: the variation in duration of therapy, differences in the baseline disease status, and variation in the doses of vortioxetine used. In support of our last assumption, the meta-regression demonstrated a larger reduction in depression symptoms as the vortioxetine dose increases. Second, during vortioxetine therapy the presence or absence of adverse events that are associated with other currently available antidepressants such as sexual dysfunction, weight change, suicide risk, cognitive impairment are not assessed in this study. Third, studies designed to assess efficacy are usually conducted with relatively smaller sample size and with shorter duration of therapy; thus, meta-analysis by including studies which were not primarily designed to assess adverse events may not have adequate power to assess rare adverse events [24]. Thus, the findings on adverse events related with vortioxetine in this study may not be accurate. Fourth, this study did not assess the possibility of correlation because of multiple dose-placebo comparisons. While, all the included studies were sponsored by pharmaceutical companies. Studies that are sponsored by pharmaceutical companies are likely to be biased by business interests.

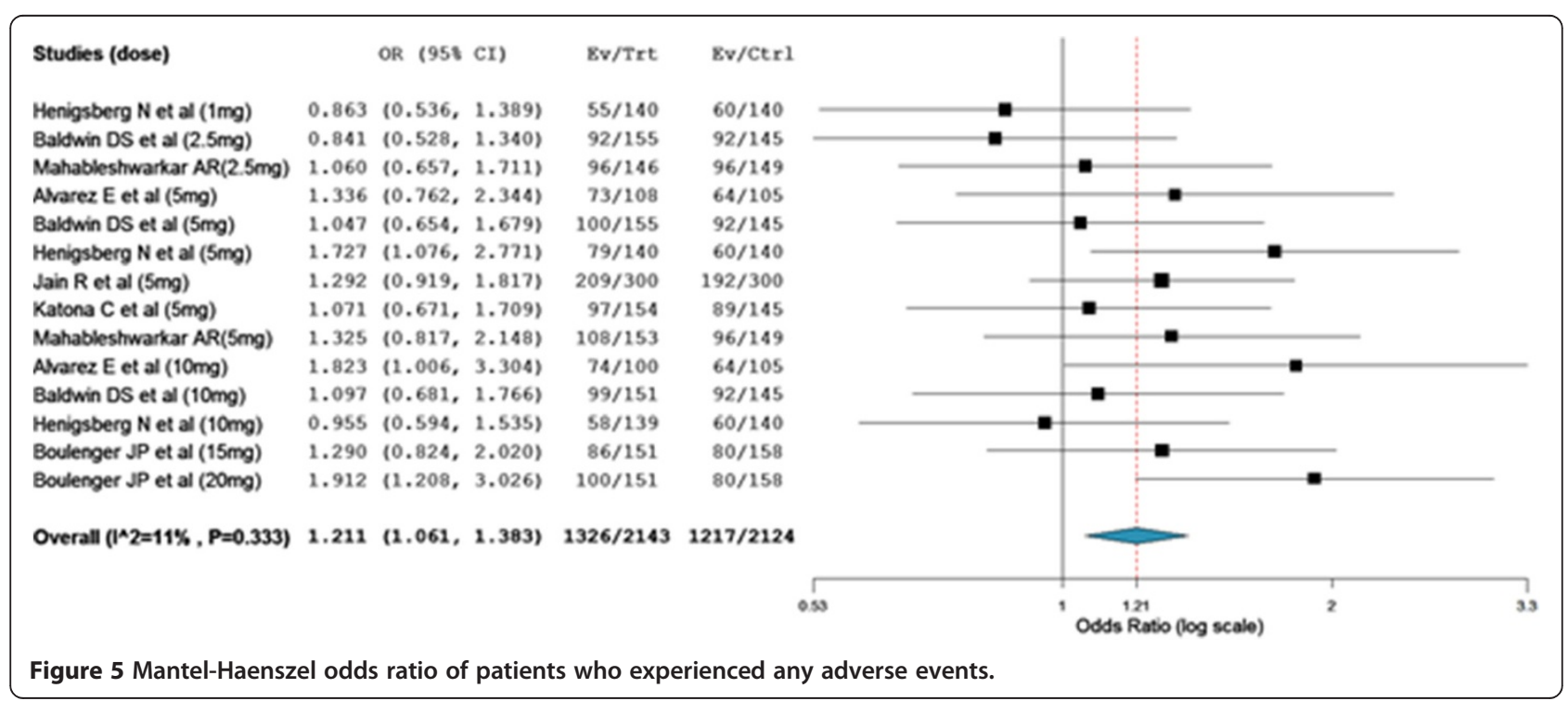




\section{Conclusions}

In conclusion vortioxetine was significantly associated with reduction in MADRS total score and HAM-D24 score from baseline. Furthermore, a statistically significant number of patients with MDD who were on vortioxetine have achieved a greater than or equal to $50 \%$ reduction in depression symptoms from baseline. Nevertheless, a significant number of patients who were on vortioxetine therapy have experienced adverse events. Thus, its long-term safety and its consistent efficacy in patients with MDD needs further investigations.

\section{Competing interests}

The authors declare that they have no competing interests.

\section{Authors' contributions}

ABe: conceived and designed the study, conducted the analysis, wrote the manuscript and participated in the literature search, study selection and data abstraction. ABa: reviewed and edited the manuscript and participated in the literature search, study selection and data abstraction. Both authors read and approved the final manuscript.

\section{Acknowledgments}

The authors would like to thank World Health Organization (WHO) and publishers in setting up HINARI. To conduct this meta-analysis the authors have not got any financial or technical support.

\section{Author details}

${ }^{1}$ Hawassa University College of Medicine and Health Sciences, P. O. Box 1560, Hawassa, Ethiopia. ² eteran Affairs Medical Center, Iron Mountain, Michigan, USA.

Received: 2 April 2014 Accepted: 23 September 2014

Published online: 27 September 2014

\section{References}

1. Pehrson AL, Sanchez C: Serotonergic modulation of glutamate neurotransmission as a strategy for treating depression and cognitive dysfunction. CNS Spectr 2014, 19(2):121-133.

2. Serretti A, Chiesa A: Treatment-emergent sexual dysfunction related to antidepressants: a meta-analysis. J Clin Psychopharmacol 2009, 29:259-266.

3. Ranjbar S, Pai NB, Deng C: The Association of Antidepressant Medication and Body Weight Gain. OJHAS 2013, 12(1):1-9.

4. Wu C, Gau SS, Lai M: Long-term antidepressant use and the risk of type 2 diabetes mellitus: a population-based, nested case-control study in Taiwan [CME]. J Clin Psychiatry 2014, 75:31-38.

5. Halperin D, Reber G: Influence of antidepressants on hemostasis. Dialogues Clin Neurosci 2007, 9(1):47-59.

6. Bang-Andersen B, Ruhland T, Jørgensen M, Smith G, Frederiksen $K$, Jensen KG, Zhong H, Nielsen SM, Hogg S, Mørk A, Stensbøl T: Discovery of 1-[2-(2,4-dimethylphenylsulfanyl)phenyl]piperazine (Lu AA21004): a novel multimodal compound for the treatment of major depressive disorder. J Med Chem 2011, 54(9):3206-3221.

7. Mork A, Pehrson A, Brennum LT, Nielsen SM, Zhong H, Lassen AB, Miller $\mathrm{S}$, Westrich L, Boyle NJ, Sanchez C, Fischer CW, Liebenberg N, Wegener G, Bundgaard C, Hogg S, Bang-Andersen B, Stensbol TB: Pharmacological effects of Lu AA21004: A novel multimodal compound for the treatment of major depressive disorder. J PHarmacol Exp Ther 2012, 340:666-675.

8. Areberg J, Søgaard B, Højer A: The clinical pharmacokinetics of Lu AA21004 and its major metabolite in healthy young volunteers. Basic Clin Pharmacol 2012, 111:198-205.

9. Chen G, Lee R, Højer A, Buchbjerg JK, Serenko M, Zhao Z: Pharmacokinetic drug interactions involving Vortioxetine (Lu AA21004), a multimodal antidepressant. Clin Drug Investig 2013, 33:727-736.

10. Alvarez E, Perez V, Dragheim M, Loft H, Artigas F: A double-blind, randomized, placebo-controlled, active reference study of Lu AA21004 in patients with major depressive disorder. Int J Neuropsychoph 2012, 15:589-600
11. Katona C, Hensen T, Olsen, Christina, Kurre, Olsen A, Christina A, Kurre A: A randomized, double-blind, placebo-controlled, duloxetine-referenced, fixed-dose study comparing the efficacy and safety of Lu AA21004 in elderly patients with major depressive disorder. Int J Neuropsychoph 2012, 27:215-223

12. Boulenger J, Loft $\mathrm{H}$, Olsen CK: Efficacy and safety of vortioxetine (Lu AA21004), 15 and 20mg/day: a randomized, double-blind, placebo-controlled, duloxetine-referenced study in the acute treatment of adult patients with major depressive disorder. Int Clin Psychopharmacol 2014, 29(3):138-149.

13. Henigsberg $N$, Mahableshwarkar AR, Jacobsen $P$, Chen $Y$, Thase ME: A randomized, double-blind, placebo-controlled 8-week trial of the efficacy and tolerablity of multiple doses of Lu AA21004 in adults with major depressive disorder. J Clin Psychiatry 2012, 73:953-959.

14. Baldwin DS, Loft H, Dragheim M: A randomised, double-blind, placebo controlled, duloxetine-referenced, fixed-dose study of three dosages of Lu AA21004 in acute treatment of major depressive disorder (MDD). Eur Neuropsychopharm 2012, 27:215-223.

15. Jain $R$, Mahableshwarkar AR, Jacobsen PL, Chen $Y$, Thase ME: $A$ randomized, double-blind, placebo-controlled 6-wk trial of the efficacy and tolerability of $5 \mathrm{mg}$ vortioxetine in adults with major depressive disorder. Int J Neuropsychoph 2013, 16:313-321.

16. Mahableshwarkar AR, Jacobsen $\mathrm{PL}$, Chen Y: A randomized, double-blind trial of $2.5 \mathrm{mg}$ and $5 \mathrm{mg}$ vortioxetine (Lu AA21004) versus placebo for 8 weeks in adults with major depressive disorder. Curr Med Res Opin 2013, 29:217-226.

17. Wallace BC, Dahabreh IJ, Trikalinos TA, Lau J, Trow P, Schmid CH: Closing the gap between methodologists and end-users: $\mathrm{R}$ as a computational back-end. J Stat Softw 2012, 49:5.

18. Alam MY, Jacobsen PL, Chen Y, Serenko M, Mahableshwarkar AR: Safety, tolerability, and efficacy of vortioxetine (Lu AA21004) in major depressive disorder: results of an open-label, flexible-dose, 52-week extension study. Int J Neuropsychoph 2014, 29:36-44.

19. Baldwin DS, Thomas H, loana F: Vortioxetine (Lu AA21004) in the long-term open-label treatment of major depressive disorder. Curr Med Res Opin 2012, 28(10):1717-1724.

20. Machado-Vieira R, Baumann J, Wheeler-Castillo C, Latov D, Henter loline D, Salvadore G, Zarate J, Carlos A: The timing of antidepressant effects: a comparison of diverse pharmacological and somatic treatments. Pharmaceuticals 2010, 3:19-41.

21. Fanelli D: How many scientists fabricate and falsify research? a systematic review and meta-analysis of survey data. PLOS ONE 2009, 4(5):4:e5738.

22. Citrome L: Vortioxetine for major depressive disorder: a systematic review of the efficacy and safety profile for this newly approved antidepressant what is the number needed to treat, number needed to harm and likelihood to be helped or harmed? Int J Clin Pract 2014, 68:60-82.

23. Theunissen EL, Street D, Højer A, Vermeeren A, Van Oers A, Ramaekers JG: A randomized trial on the acute and steady-state effects of a new antidepressant, Vortioxetine (Lu AA21004), on actual driving and cognition. Clin Pharmacol Ther 2013, 93:493-501.

24. Walker E, Hernandez AV, Kattan MW: Meta-analysis: its strengths and limitations. Clev Clin J Med 2008, 75:431-439.

\section{doi:10.1186/s12888-014-0276-x}

Cite this article as: Berhan and Barker: Vortioxetine in the treatment of adult patients with major depressive disorder: a meta-analysis of randomized double-blind controlled trials. BMC Psychiatry 2014 14:276. 